\title{
On the heterogeneity of local shear strain induced by high-pressure torsion
}

Wei Jiang, Hao Zhou, Jinfeng Nie, Yang Cao, * Yusheng Li, Xiaozhou Liao, Yonghao Zhao, Megumi Kawasaki, Terence G Langdon, Yuntian Zhu*

Prof. Y.T. Zhu, Prof. Y.H. Zhao, Dr. Y. Cao, Dr. H. Zhou, Dr. J.F. Nie, Dr. Y.S. Li, W. Jiang,

Nano and Heterogeneous Materials Center, School of Materials Science and Engineering, Nanjing University of Science and Technology, Nanjing 210094, P.R. China

E-mails: y.cao@njust.edu.cn (Y. Cao); ytzhu@ncsu.edu (Y.T. Zhu)

Prof. X.Z. Liao

School of Aerospace, Mechanical and Mechatronic Engineering, The University of Sydney, Sydney, NSW 2006, Australia

Prof. M. Kawasaki

School of Mechanical, Industrial and Manufacturing Engineering, Oregon State University, Corvallis OR 97331-6001, USA

Prof. T.G. Langdon

Materials Research Group, Department of Mechanical Engineering, University of Southampton, Southampton SO17 1BJ, UK

Prof. Y.T. Zhu

Department of Materials Science and Engineering, North Carolina State University, Raleigh, NC 27695, USA

\begin{abstract}
A ductile duplex stainless steel consisting of a ferrite phase and an austenitic phase was chosen as a model material to investigate the development of shear strain patterns under high-pressure torsion. Systematic analysis on the macroscopic and microscopic heterogeneities of local shear strain revealed that complex shear patterns can be developed only above a high strain level where the grain sizes have already been refined to a steady-state. It is concluded that grain boundary mediated deformation mechanisms provide sufficient freedom for reshaping the austenite phase domains and thus the intrinsic factor required for the formation of these complicated shear patterns is well-developed ultrafine grains and/or nano-grains.
\end{abstract}




\section{Introduction}

High-pressure torsion (HPT) is so far the most effective SPD method for processing bulk nanostructured materials. ${ }^{[1]}$ After 30 years of research and development in this field, researchers have achieved substantial understanding about this method and the corresponding effects on the microstructures and mechanical properties of materials. ${ }^{[2-4]}$

The principle of quasi-constrained HPT is illustrated schematically in Figure 1a. A disk-shaped sample is placed between two massive anvils and subjected to an applied pressure $\mathrm{P}$ in the range between a few hundred MPa to a few tens of GPa. Then torsional strain is imposed by the rotation of either the lower or the upper anvil. The compressive load/pressure imposed on the disk, which is constrained in the cavity between the upper and lower anvils, is effective in preventing cracking of the sample. The torsion provided by the rotating anvil is theoretically capable of imposing unlimited strain to the sample. Figure 1b shows a three-dimensional reconstruction of a half HPT disk, which is a simple three-dimensional drawing based on the optical images taken from the exposed surfaces of a half 10-revolutions HPT disk. The HPT disk would eventually deform to a shape that fits well to the cavity between the anvils. The rough surface of the cavity provides excellent grip to the disk but leaves marks on the disk surface. The high strain imposed by HPT causes noteworthy microstructural evolution to the sample material and this is revealed on the polished cross-sections of the disk.

There are also unconstrained and constrained HPT setups and these are described in more detail elsewhere. ${ }^{[1,5,6]}$ Despite the different HPT setups, the basic principle of HPT is the same so that the equivalent von Mises strain imposed on the HPT disk is given by a relationship:

$\varepsilon_{e q}=\frac{2 \pi N r}{h \sqrt{3}}$

where $\mathrm{N}$ is the number of HPT revolutions, $\mathrm{r}$ is the radial distance from the center of the disk, and h is the disk thickness. Eq. (1) indicates that there will always be a strain gradient with low strain at the disk center and high strain the disk periphery. Eq. (1) also indicates that the shear strain at the same radial distance to the center will be the same. However, there have been numerous reports showing that the actual shear strain imposed by HPT may deviate significantly from the ideal situation described by Eq. (1) ${ }^{[7-10]}$ and these results demonstrate hardly predictable heterogeneity throughout the disk sample. The early discovery of the heterogeneity of local shear strain induced by HPT was by means of mapping the hardness throughout the surface of an HPT disk ${ }^{[7}$, ${ }^{11]}$ but the indirect measurement of shear strain was not able to fully explain the phenomenon. Nature abounds with visible patterns of various kinds as does the shear strain. In 2010, Cao et al. ${ }^{[10,12,13]}$ proposed a method of visualizing shear strain by using ductile dual phase materials with easily distinguishable phase domain structures. Since then, the study on the shear strain imposed by HPT became much easier ${ }^{[5,14-16]}$ and it was quickly realized that the heterogeneity of local shear strain was a result of 
local shear turbulence, shear vortices and shear bands. ${ }^{[10,13,14]}$

Macroscopic shear strain heterogeneity can be well explained by the existence of bifurcations under plastic flow including shear turbulence, shear vortices and shear bands. However, there is also the microscopic shear strain heterogeneity, which is inherent to the characteristics of crystal structures and slip systems. For example, an allotropic phase transformation during deformation may cause heterogeneity in local shear strain ${ }^{[17,18]}$ and the asymmetric natures of twinning and slip in polycrystalline FCC and HCP materials may cause heterogeneities in local shear strain. ${ }^{[4,19]}$ In contrast to the macroscopic shear strain heterogeneity, which is revealed as flow patterns of irregular shapes, the microscopic shear strain heterogeneity is revealed as phase boundaries and domain boundaries of irregular shapes. The nanostructured materials processed by SPD are often referred to as interface-controlled materials because the spatial arrangements of grain boundaries, phase boundaries and domain boundaries predominantly govern the mechanical properties of these materials ${ }^{[20-22]}$. Thus, the microscopic heterogeneity of local shear strain imposed by HPT is obviously another fundamental issue in mastering the HPT process.

Despite the fact that the macroscopic shear strain heterogeneity and the microscopic shear strain heterogeneity are equally important in the basic principles of the HPT processing of metallic materials, there is no research investigating the correlation between the heterogeneities of different scales. Thus, the current work examines both the macroscopic shear strain heterogeneity and the microscopic shear strain heterogeneity introduced by the HPT processing of duplex stainless steels. The relationship between the heterogeneities of different scales is explained in detail based on the macrostructural and microstructural evolution of the materials with increasing strain.

\section{Experimental materials and procedures}

A commercial DP3W duplex stainless steel containing approximately equal volume fractions of the bcc ferrite ( $\alpha$ ) phase and the fcc austenite $(\gamma)$ phase were used in this investigation. For additional details of the DP3W duplex stainless steel, readers are referred to relevant literatures. ${ }^{[13,23,24]}$ Disks with a diameter of $\sim 9.8 \mathrm{~mm}$ and a thickness of $\sim 1.7 \mathrm{~mm}$ were cut from the steel plates. Both sides of each disk were polished using abrasive papers (180 to 4000 grit) to make a series of disk samples having final thicknesses of $0.79-0.80 \mathrm{~mm}$ ready for HPT processing. The HPT experiments were conducted under quasi-constrained conditions with a rotational speed of 1 RPM. 14 disks were processed under 6 GPa pressure for 1, 2, 3, 5, 6, 10 and 16 revolutions, and two disks were processed under $8 \mathrm{GPa}$ pressure for 16

revolutions. Based on our experimental observation and available literature, ${ }^{[25,26]}$ slippage in HPT processing is minimized with an applied pressure above $3 \mathrm{GPa}$. There was no recorded slippage for samples processed less than 5 revolution in these experiments. The reason for using the quasi-constrained HPT method is that it overcomes the drawbacks of sample thinning and increased torque when processing using unconstrained and fully constrained HPT methods, respectively. ${ }^{[27]}$ 
Optical microscopy and transmission electron microscopy (TEM) analysis were conducted on the as-received and HPT samples to help in revealing the nature of the heterogeneity of the local shear strain imposed by HPT. In order to achieve good contrast between the $\alpha$-phase and $\gamma$-phase under optical microscopy, several different methods were evaluated for preparing the material surface. It was eventually found that there are two methods that lead to promising results: (1) by using a Struers OP-U-0.04 $\mu \mathrm{m}$ suspension in the final polishing stage, consistent but light contrast between the two phases was revealed throughout the entire polished surface. (2) chemical etching by a solution of $45 \%$ water, $30 \%$ nitric acid, $10 \%$ hydrofluoric acid and $15 \%$ hydrochloric acid significantly improved the contrast between the two phases but the result were inconsistent and they were affected by the etching time, temperature and humidity. Extensive optical microscopy (OM) analysis was conducted with an Olympus BX-61 optical microscope and a ZEISS-Axio-Vert-A1 optical microscope. Each TEM sample was taken parallel to the disk surface, and prepared by electro-polishing using a Struers TenuPol-5 jet electro-polishing unit and a solution of $23 \%$ perchloric acid and $77 \%$ acetic acid, under an operating voltage of $20 \mathrm{~V}$ and at a temperature of $20{ }^{\circ} \mathrm{C}$ until a central perforation surrounded by a thin area was formed. Careful control of the electro-polishing condition can guarantee a nearly $100 \%$ success rate and excellent sample quality. TEM characterization was performed with a Philips CM12 microscope operating at $120 \mathrm{kV}$ and a TECNAI G2 20 microscope operating at $200 \mathrm{kV}$.

\section{Results}

The commercial DP3W duplex stainless steel has equal volume fractions of $\propto$-phase and $\gamma$-phase. Both phases are ductile and easily recognizable under an optical microscope and SEM, thereby making the material ideal for visualization of shear strain. As shown in Figure 2, the $\alpha$-phase matrix appears in dark contrast and the $\gamma$-phase domains appear in light contrast under an optical microscope. A three-dimensional reconstruction of the structure shows that the $\gamma$-phase domains have lengths of 100-1000 $\mu \mathrm{m}$ and an elliptical cross-section with long widths of 10-100 $\mu \mathrm{m}$ and short widths of 5-50 $\mu \mathrm{m}$. By increasing HPT strain, the shapes and orientations of the $\gamma$-phase domains are gradually changed. As shown in Figure 3a, when the disk was processed to 3 revolutions under 6 GPa pressure, the majority of the $\gamma$-phase domains tend to reorient towards the direction of the HPT rotation. 
However, in general the shear strain pattern appears chaotic and inhomogeneous. When the disk was processed to 16 revolutions, the shear strain pattern was very close to the ideal situation in which the majority of the phase domains are parallel to the direction of the HPT-imposed strain (parallel to the circumference of the disk). However, in this particular case, shear turbulence and shear vortices are still abundant in the regions approximately $1 \mathrm{~mm}$ from the disk circumference. This is due to the gradient back-pressure experienced at the circumference of the disk and the anvil misalignmen. ${ }^{[14]}$

There are numerous results showing that macroscopic heterogeneity is evident prior to reaching homogeneous microstructures. In the case of high strength materials such as steels, macroscopic heterogeneity is abundant when samples are processed to 3 to 5 revolutions. ${ }^{[12-14]}$ The major features of local shear heterogeneities are sharp shear bands as marked by red arrows in Figure 4, shear turbulence shown in Figure 4c, and shear vortices shown in Figure 4d, where Figure 4a-b were taken at the edge of the disk, and Figure c-d were taken approximately $1.5 \mathrm{~mm}$ away from the edge of the disk. Shear bands are mostly observed at the edge of the disks, but shear turbulence and shear vortices may exist at any location on the disk surface except the disk center. Shear bands are very effective in causing distortion to local shear strain patterns and fragmentation to the local microstructures, ${ }^{[28,29]}$ indicated by the sharp changes in shear patterns on both sides of the shear bands as shown in Figure 4.

The heterogeneity induced by HPT processing is also examined at the microscale. At this level the local shear strain is reflected by the shape of the grains and phase domains, and by the trace of the grain boundaries and phase boundaries. For comparison, the microstructures of the as-received steel samples were examined first. As shown in Figure 5, the $\alpha$-phase and the $\gamma$-phase appear in light and dark contrast, respectively, because the $\gamma$-phase in the steel contains a large proportion of $\mathrm{Ni}$ atoms which are much more effective in scattering electrons than Fe atoms. Diffraction patterns in Figure $5 \mathrm{~b}$ and $\mathrm{c}$ are taken from the grains labelled $\alpha$ and $\gamma$, respectively, to justify the crystal structures of the $\alpha$-phase and the $\gamma$-phase. The grain boundaries and phase boundaries in the as-received samples are smooth and sharp. Dislocation densities in both phases are low, indicating very low strain and low residual stresses in both phases.

As shown in Figure 6, where the theoretical von Mises strain is in the range of 2 - 5 (near the center of 1 revolution HPT disk), the austenite grain is featured with a high density of nano-twins, and the $\alpha$-phase region is featured with ultrafine sub-grains. In this case, the interface between the $\alpha$-phase and the $\gamma$-phase is also the grain boundary of the austenite grain. The austenite grain boundary is no longer smooth in contrast to the grain boundaries in the as-received sample (Figure 5). Numerous twin boundaries attached to the grain boundary indicate that deformation twinning is a major contributor to the shear strain experienced by the austenite grain. ${ }^{[30-32]}$ It is worth noting that dislocation slip also plays an important role in accommodating the shear strain in the austenite grain but the freedom of dislocation motion is strongly restricted by the twin boundaries. ${ }^{[33]}$ On the other hand, three-dimensional dislocation structures were formed in the accommodating shear 
strain in the $\alpha$-phase matrix, granting the $\alpha$-phase matrix the freedom of changing to any complex shape.

As the equivalent von Mises strain increased to the range of $5-20$, the $\gamma$-phase close to the center of the steel disk possesses a mixture of ultrafine sub-grains and fragmented twin bundles, as shown in Figure 7. The grain containing a high density of nano-twins was sliced into a few parallel pieces by the white bands. The angle between the white bands and the $\{111\}$ twinning plane is approximately $70^{\circ}$. An early report has shown that parallel primary twins could lose coherency and become thin band structures with an angle of $60^{\circ}-70^{\circ}$ to a $\{111\}$ twinning plane under increased shear strain. ${ }^{[23,34]}$ In contrast, the angle between the $\{111\}$ twinning planes and a microscopic shear band is usually much smaller in the range of $20-40^{\circ} .{ }^{[34,35]}$ Therefore, the white bands were primary twins which lost coherency after interaction with secondary twins and dislocations. It is worth mentioning that microscopic shear bands have been observed in some materials with low stacking fault energies processed by HPT. ${ }^{[36]}$ However, microscopic shear bands were not observed in HPT processed duplex stainless steels. ${ }^{[23]}$ Thus, the shear bands discussed in this work are macroscopic shear bands, which are readily observable under an optical microscope in many materials processed by HPT. ${ }^{[13,37,38]}$

When sufficient shear strains are imposed to the disk samples, both $\alpha$-phase and

$\gamma$-phase can be refined to nanostructures. Figures 8 shows the microstructures of the periphery regions of an HPT disk processed to 5 revolutions under 6 GPa pressure. The equivalent von Mises strain in the local region was estimated to be in the range of $60-100$. The sizes of the ferrite grains are in the ultrafine-grained regimes and the sizes of the austenite grains are mostly in the nano-crystalline regime. The $\gamma$-phase domains may possess a very complex shape as shown in Figure 8a and a small volume of ferrite grains was partially squeezed into the $\gamma$-phase domain in this particular region thereby indicating a very complicated strain condition in the local area. As shown in Figure 8 b and c, the interphase interfaces vary significantly from one area to another. The ultrafine grain sizes and active grain boundaries grant the freedom for phase domains and interphase interfaces to adapt to any shapes.

Figure 9 shows high-resolution TEM images of a typical $\gamma$-grain and $\alpha$-grain.

Figure 9a shows a $\gamma$-grain that contains many stacking faults. Every stacking fault has at least one end attached to the grain boundary. Figure $9 \mathrm{~b}$ is an enlarged image of the selected area in Figure 9a. The longest extrinsic stacking fault in the grain, shown in Figure 9a, has one end attached to the grain boundary shown in Figure 9b. The extrinsic stacking fault shifted the atoms on two adjacent atomic planes away from the original positions of a perfect lattice structure. Figure 9c shows an $\alpha$-grain that has most of the dislocations located close to the grain boundary as shown in Figure 9d.

\section{Discussion}


According to Eq. 1, the shear strain imposed by HPT will gradually shear and twist each unit volume in the sample. If each unit volume is in the shape of a cube, the shear strain imposed by HPT causes the top surface of the cube to shear and gives a simultaneous shift with respect to the bottom surface. A detailed description of the ideal shear strain imposed by HPT is given elsewhere. ${ }^{[1,39,40]}$ However, deviations from the ideal situation are always encountered in real practice, resulting in the heterogeneity of local shear strain. Macroscopic shear strain heterogeneity in the form of shear turbulence, shear vortices and shear bands, as shown in Figure 3 and Figure 4, clearly reveal the complexities of the local shear strain. Thus, the major outstanding issue is to determine the types of deformation mechanism that are capable of the strain accommodation.

In the case of crystalline materials, dislocation slip and/or deformation twinning are common strain accommodation mechanisms. However, both dislocation slip and deformation twinning have directionalities, which are strictly limited by the available slip systems and twinning systems, respectively. In the current case, the $\alpha$-phase was deformed by dislocation slip and the $\gamma$-phase was deformed by both dislocation slip and twinning, as revealed by comparing Figure 5 and Figure 6. As shown in Figure 6, the $\alpha$-phase domain contains dislocation substructures that tend to elongate in the vertical direction. This indicates that the local shear strain was roughly in the vertical direction. ${ }^{[40]}$ In contrast, the $\gamma$-phase was deformed mainly by twinning due to the low stacking fault energy and high imposed pressure/stress. ${ }^{[33,41]}$ The coherent twin boundaries have an acute angle to the direction of elongation of the dislocation substructures in the $\alpha$-phase, indicating that the local shear stress favors the twining shear. High densities of deformation twins and geometrically necessary dislocations at the phase boundaries are capable of accommodating the small but complex local shear strain that leads to wavy phase/grain boundaries.

There is a critical issue that once an austenite grain was filled with a high density of deformation twins as shown in Figure 6 the grain then possesses an apparent flow stress anisotropy because dislocation slip and twinning are much easier in directions parallel to the coherent boundaries of the high-density nano-twins than in other directions. ${ }^{[4,42]}$ As shown in Figure 7, the austenite grain has been "sliced" into a few pieces by white bands. The white bands have an angle of $\sim 70^{\circ}$ to the coherent twin boundaries, indicating that the white bands are deformation twins of another twinning system that have lost coherency due to strain localization. This grain can be sheared in directions parallel to the white bands but hardly changes its shape in other ways. Thus, it is obvious that this austenite grain is incapable of accommodating a large strain such as a shear vortex. By contrast, as the grain sizes are reduced to the ultrafine-grained regime and lower, the $\gamma$-phase domains possess very complex shapes as shown in Figure 8. For the duplex steel used in the current experiment, a shear strain of above 20 was necessary in order to achieve substantial grain refinement to the ultrafine-grained regime. Consider the fact that macroscopic shear vortices and shear bands were only observed in the HPT disks processed to three and more revolutions and in the regions where the local von Mises strains were estimated to be larger than 40. Thus, a localized large shear strain in the form of shear vortices and 
shear bands can only be accommodated with the assistance of grain boundary mediated processes that are characteristic deformation mechanisms of ultrafine grains and nano-grains. ${ }^{[4]}$ Figure 9 provides solid evidence of grain boundary mediated plasticity in the nanocrystalline duplex stainless steel processed by HPT to 5 revolutions. As shown in Figure 9a and b, large numbers of stacking faults were formed in the $\gamma$-grain by the emission of partial dislocations from the grain boundary. As shown in Figure 9c and d, the majority of the dislocations in an $\alpha$-grain are located close to the grain boundary, but in the interior of the grains the dislocation density is low. Thus, the plastic strain at the edge of the 5 revolutions HPT sample is mainly accommodated by grain boundary mediated deformation mechanisms. ${ }^{[8,44-48]}$ There are reports showing that at the hardening stage of HPT processing the major work is performed in causing grain refinement. ${ }^{[49]}$ Once the grain sizes reach the new steady-state, a further increase in strain would be mainly accommodated by dynamic recovery, ${ }^{[8]}$ grain rotation ${ }^{[44,45]}$ and grain boundary sliding. ${ }^{[46,47]}$ These grain boundary mediated activities are effective in redistributing and reshaping the phase domains. ${ }^{[49]}$

The shear strain carried by shear turbulence is complex but not necessarily high, and it is strongly related to the initial shapes and orientations of the $\gamma$-phase domains. As shown in Figure 7, the irregularity of the austenite grain has sizes that are comparable to shear turbulence patterns at low strain levels which were observed near the centers of two and three revolutions HPT samples in earlier work. ${ }^{[12,13]}$ In addition, the stream-like shear turbulences shown in Figure $4 c$ and $d$ are very thin and interweaved with other shear structures such as shear bands and shear vortices. This indicates that shear turbulence in the high strain region and in the low strain region may be accommodated by different deformation mechanisms.

There are numerous reports showing that when the imposed shear strain is sufficiently high the shear strain patterns throughout the disk become comparable to the ideal situation. ${ }^{[10,13,39]}$ However, as shown in Figure 3b, a ring-shaped region featuring shear instability outlined the periphery of the 16-revolution disk. This indicates that even at a very high strain level the ideal shear strain patterns may not develop because the top and bottom anvils of the HPT facility may be misaligned by approximately $100 \mu \mathrm{m}$ or larger. ${ }^{[14]}$ In fact, the major experimental factor that causes shear strain instability including shear bands, shear vortices and even double shear swirls $^{[13]}$ is anvil misalignment. ${ }^{[14]}$

\section{Conclusions}

Macroscopic heterogeneity induced by HPT was revealed by the complex shear strain patterns including shear bands, shear vortices and shear turbulences. While shear turbulence was observed in nearly all strain levels, shear bands and shear vortices were found only at high strain levels where the grain refinement had already reached the steady-state.

Microscopic local shear strain heterogeneities were revealed by shapes of the grains and phase domains and by the traces of the grain boundaries and phase boundaries. At low strain levels, when the grain sizes are in the scale of micrometers, dislocation slip and deformation twinning accommodates the local shear strain. Thus, 
the shapes of the grains and phase domains are determined by the active slip systems and twinning systems. The freedom for changing the shapes of grains and phase domains are then very limited. Therefore, complex shear patterns such as macroscopic shear bands and shear vortices cannot form at these low strain levels.

In the current work, it is proven that the intrinsic factor required for the formation of complex shear patterns is well-developed ultrafine grains and/or nano-grains. Once the grains are refined to the ultrafine-grained regime and the nano-crystalline regime, further shear straining is mainly accommodated by grain boundary mediated mechanisms including grain rotation and grain boundary sliding. These grain boundary mediated activities are effective in redistributing and reshaping the phase domains, thus allowing the formation of macroscopic shear bands and shear vortices.

\section{Acknowledgements}

This work was supported by the National Natural Science Foundation of China (51601094 (Y.C.)), the National Key R\&D Program of China (2017YFA0204403), the Fundamental Research Funds for the Central Universities (30918011342 (H.Z.) and 30919011412 (Y.T.Z.)) and the Australian Research Council (DP150101121). This work was also supported in part by the National Science Foundation of the United States (DMR-1810343 (M.K.)) and the European Research Council (ERC Grant 267464-SPDMETALS (T.G.L.)). The authors are thankful for the technical support from the Jiangsu Key Laboratory of Advanced Micro\&Nano Materials and Technology, and the Materials Characterization Facility of Nanjing University of Science and Technology.

\section{Conflict of Interest}

The authors declare no conflict of interest.

\section{Keywords}

Shear strain, High-pressure torsion, Steel, Deformation twinning, Microstructure

\section{References:}

[1] A. P. Zhilyaev, T. G. Langdon, Prog. Mater. Sci. 2008, 53, 893.

[2] T. G. Langdon, Acta Mater. 2013, 61, 7035.

[3] R. Z. Valiev, Y. Estrin, Z. Horita, T. G. Langdon, M. J. Zehetbauer, Y. T. Zhu, Mater. Res. Lett. 2016, 4, 1.

[4] Y. Cao, S. Ni, X. Liao, M. Song, Y. Zhu, Materials Science and Engineering: R: Reports 2018, 133, 1.

[5] K. S. Kormout, R. Pippan, A. Bachmaier, Advanced Engineering Materials 2017, 19, 19.

[6] R. B. Figueiredo, P. H. R. Pereira, M. T. P. Aguilar, P. R. Cetlin, T. G. Langdon, Acta Mater. 2012, 60, 3190.

[7] A. P. Zhilyaev, G. V. Nurislamova, B. K. Kim, M. D. Baró, J. A. Szpunar, T. G. Langdon, Acta Mater. 2003, 51, 753. 
[8] C. Xu, Z. Horita, T. G. Langdon, Acta Mater. 2008, 56, 5168.

[9] M. Kawasaki, B. Ahn, T. G. Langdon, Acta Mater. 2010, 58, 919.

[10]Y. Cao, M. Kawasaki, Y. B. Wang, S. N. Alhajeri, X. Z. Liao, W. L. Zheng, S. P. Ringer, Y. T. Zhu, T. G. Langdon, J. Mater. Sci. 2010, 45, 4545.

[11]C. Xu, Z. Horita, T. G. Langdon, Acta Mater. 2007, 55, 203.

[12]Y. Cao, Y. B. Wang, S. N. Alhajeri, X. Z. Liao, W. L. Zheng, S. P. Ringer, T. G. Langdon, Y. T. Zhu, J. Mater. Sci. 2010, 45, 765.

[13]Y. Cao, Y. B. Wang, R. B. Figueiredo, L. Chang, X. Z. Liao, M. Kawasaki, W. L. Zheng, S. P. Ringer, T. G. Langdon, Y. T. Zhu, Acta Mater. 2011, 59, 3903.

[14]H. Yi, K. Megumi, T. G. Langdon, Adv. Eng. Mater. 2013, 15, 747.

[15]Y. Z. Tian, S. D. Wu, Z. F. Zhang, R. B. Figueiredo, N. Gao, T. G. Langdon, Acta Mater. 2011, 59, 2783.

[16]J.-K. Han, D. K. Han, G. Y. Liang, J.-I. Jang, T. G. Langdon, M. Kawasaki, Adv. Eng. Mater. 2018, 20.

[17]A. P. Zhilyaev, F. Gálvez, A. Sharafutdinov, M. T. Pérez-Prado, Mater. Sci. Eng.A 2010, 527, 3918.

[18]Y. B. Wang, Y. H. Zhao, Q. Lian, X. Z. Liao, R. Z. Valiev, S. P. Ringer, Y. T. Zhu, E. J. Lavernia, Scr. Mater. 2010, 63, 613.

[19]Y. B. Wang, M. Louie, Y. Cao, X. Z. Liao, H. J. Li, S. P. Ringer, Y. T. Zhu, Scr. Mater. 2010, 62, 214.

[20]R. Valiev, Y. Estrin, Z. Horita, T. Langdon, M. Zehetbauer, Y. Zhu, Mater. Res. Lett. 2016, 4, 1.

[21]Y. Zhu, X. Wu, Mater. Res. Lett. 2019, 7, 393.

[22]X. An, S. Zhu, Y. Cao, M. Kawasaki, X. Liao, S. Ringer, J. Nie, T. Langdon, Y. Zhu, Appl. Phys. Lett. 2015, 107, 011901.

[23]Y. Cao, Y. B. Wang, X. H. An, X. Z. Liao, M. Kawasaki, S. P. Ringer, T. G. Langdon, Y. T. Zhu, Acta Mater. 2014, 63, 16.

[24]S. K. Samudrala, P. J. Felfer, V. J. Araullo-Peters, Y. Cao, X. Z. Liao, J. M. Cairney, Ultramicroscopy 2013, 132, 158.

[25]K. Edalati, Z. Horita, T. G. Langdon, Scr. Mater. 2009, 60, 9.

[26]M. Kamrani, V. I. Levitas, B. Feng, Mater. Sci. Eng.A 2017, 705, 219.

[27]R. B. Figueiredo, P. R. Cetlin, T. G. Langdon, Mater. Sci. Eng., A 2012, 528, 8198.

[28]F. J. Humphreys, M. Hatherly, in Recrystallization and Related Annealing Phenomena (Second Edition), (Eds: F. J. Humphreys, M. Hatherly), Elsevier, Oxford 2004, 11.

[29]S. D. Antolovich, R. W. Armstrong, Progress in Materials Science 2014, 59, 1.

[30]X. L. Ma, H. Zhou, J. Narayan, Y. T. Zhu, Scr. Mater. 2015, 109, 89.

[31]G. Ji, L. Zhang, N. Song, S. Min, Scr. Mater. 2016, 125, 49.

[32]L. Liu, J. Wang, S. K. Gong, S. X. Mao, Phys Rev Lett 2011, 106, 175504.

[33]Y. Cao, Y. B. Wang, X. Z. Liao, M. Kawasaki, S. P. Ringer, T. G. Langdon, Y. T. Zhu, Appl. Phys. Lett. 2012, 101.

[34]H. Paul, A. Morawiec, J. H. Driver, E. Bouzy, Int. J. Plast. 2009, 25, 1588.

[35]X. H. An, S. D. Wu, Z. G. Wang, Z. F. Zhang, Prog. Mater. Sci. 2019, 101, 1. 
[36]X. H. An, Q. Y. Lin, S. D. Wu, Z. F. Zhang, R. B. Figueiredo, N. Gao, T. G. Langdon, Philosophical Magazine 2011, 91, 3307.

[37]Y. Z. Tian, X. H. An, S. D. Wu, Z. F. Zhang, R. B. Figueiredo, N. Gao, T. G. Langdon, Scr. Mater. 2010, 63, 65.

[38]Y. Huang, M. Kawasaki, T. G. Langdon, Advanced Engineering Materials 2013, 15, 747.

[39]M. Hafok, R. Pippan, Scr. Mater. 2007, 56, 757.

[40]M. Hafok, R. Pippan, Philos. Mag. 2008, 88, 1857.

[41]Y. Cao, Y. B. Wang, X. H. An, X. Z. Liao, M. Kawasaki, S. P. Ringer, T. G. Langdon, Y. T. Zhu, Scr. Mater. 2015, 100, 98.

[42]L. Lu, X. Chen, X. Huang, K. Lu, Sci. 2009, 323, 607.

[43]Z. Shan, E. A. Stach, J. M. K. Wiezorek, J. A. Knapp, D. M. Follstaedt, S. X. Mao, Sci. 2004, 305, 654.

[44]L. Wang, J. Teng, P. Liu, A. Hirata, E. Ma, Z. Zhang, M. Chen, X. Han, Nature communications 2014, 5, 4402.

[45]Y. Wang, B. Li, M. Sui, S. Mao, Appl. Phys. Lett. 2008, 92, 011903.

[46]M. Y. Gutkin, I. Ovid’Ko, N. Skiba, Acta Mater. 2003, 51, 4059.

[47]X. Li, Y. Wei, W. Yang, H. Gao, Proc. Natl. Acad. Sci. 2009, 106, 16108.

[48]L. Wang, X. Han, P. Liu, Y. Yue, Z. Zhang, E. Ma, Physical review letters 2010, 105, 135501.

[49]Y. Liu, F. Wang, Y. Cao, J. Nie, H. Zhou, H. Yang, X. Liu, X. An, X. Liao, Y. Zhao, Y. Zhu, Scr. Mater. 2019, 162, 316. 

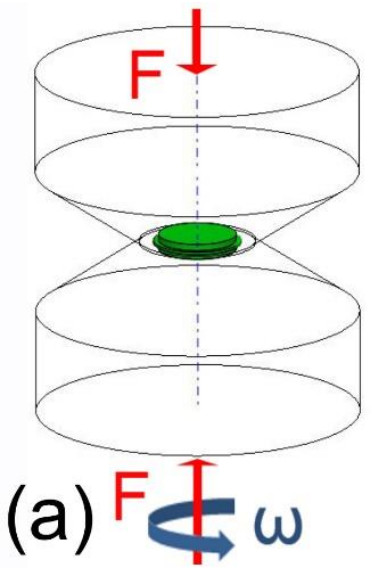

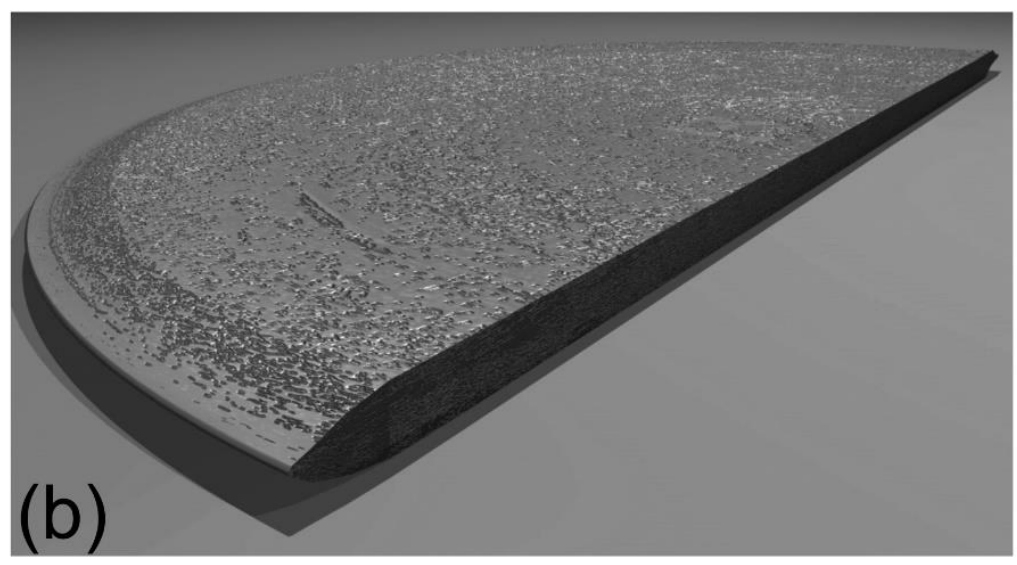

Fig. 1. (a) A Schematic illustration of the high-pressure torsion process; (b) A three-dimensional reconstruction of a half HPT disk where the morphology of the top surface and the microstructure on the cross-sectional face are revealed. 


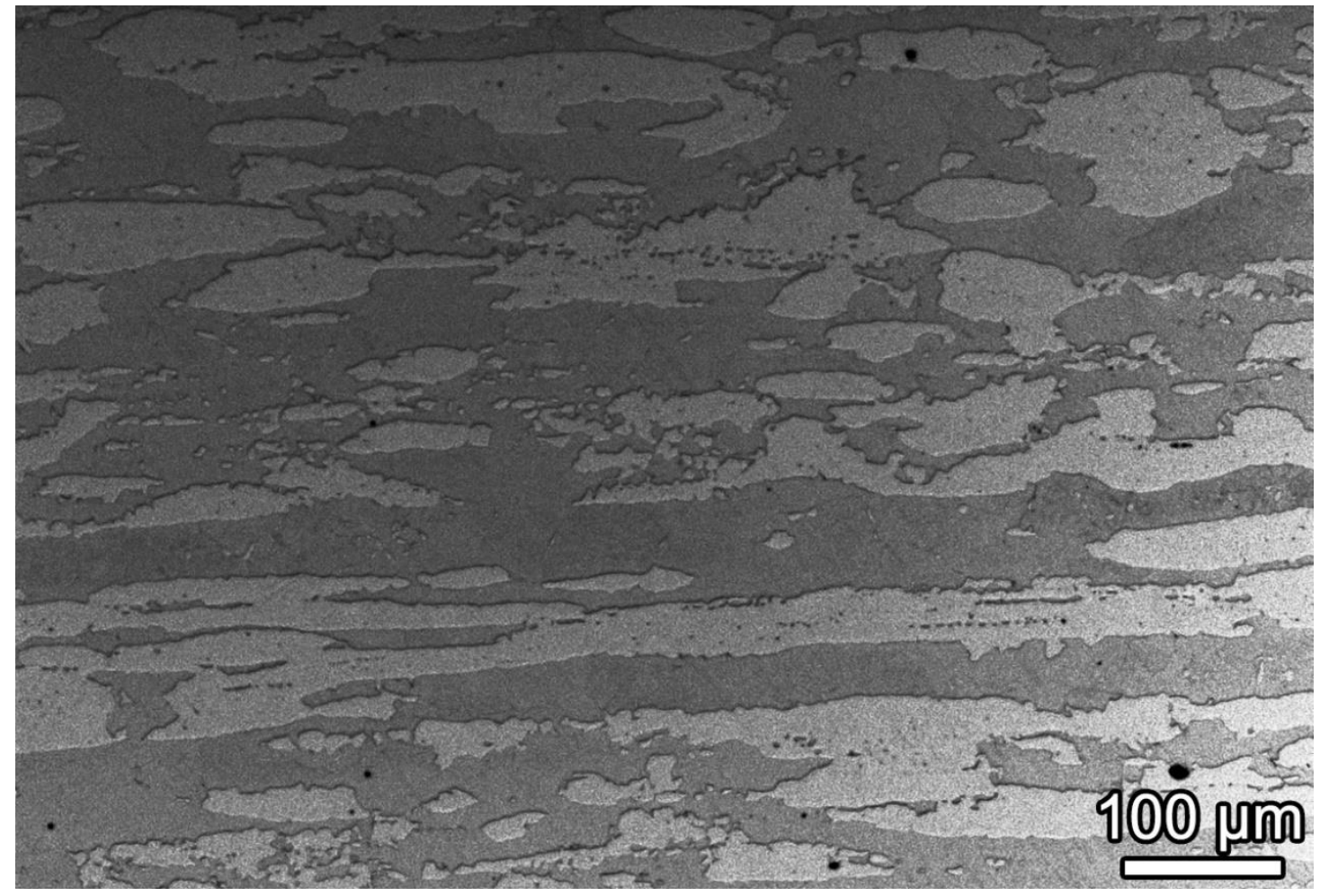

Fig. 2. An optical micrograph showing lamellar austenite phase domains embedded in the dark contrast ferrite matrix. 

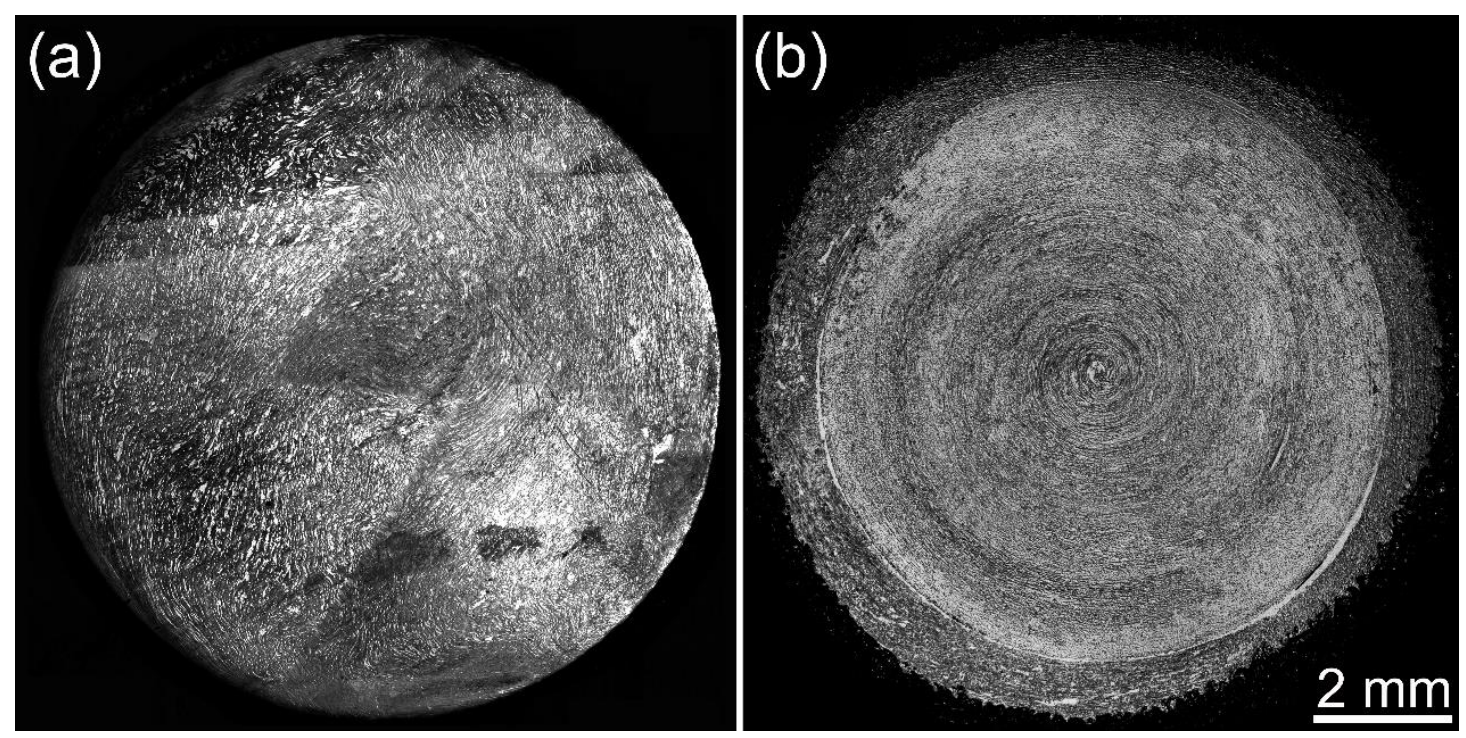

Fig. 3. Optical micrographs showing top surfaces of HPT disks processed (a) to 3 revolutions under 6 GPa pressure and (b) to 16 revolutions under 8 GPa pressure, respectively. 

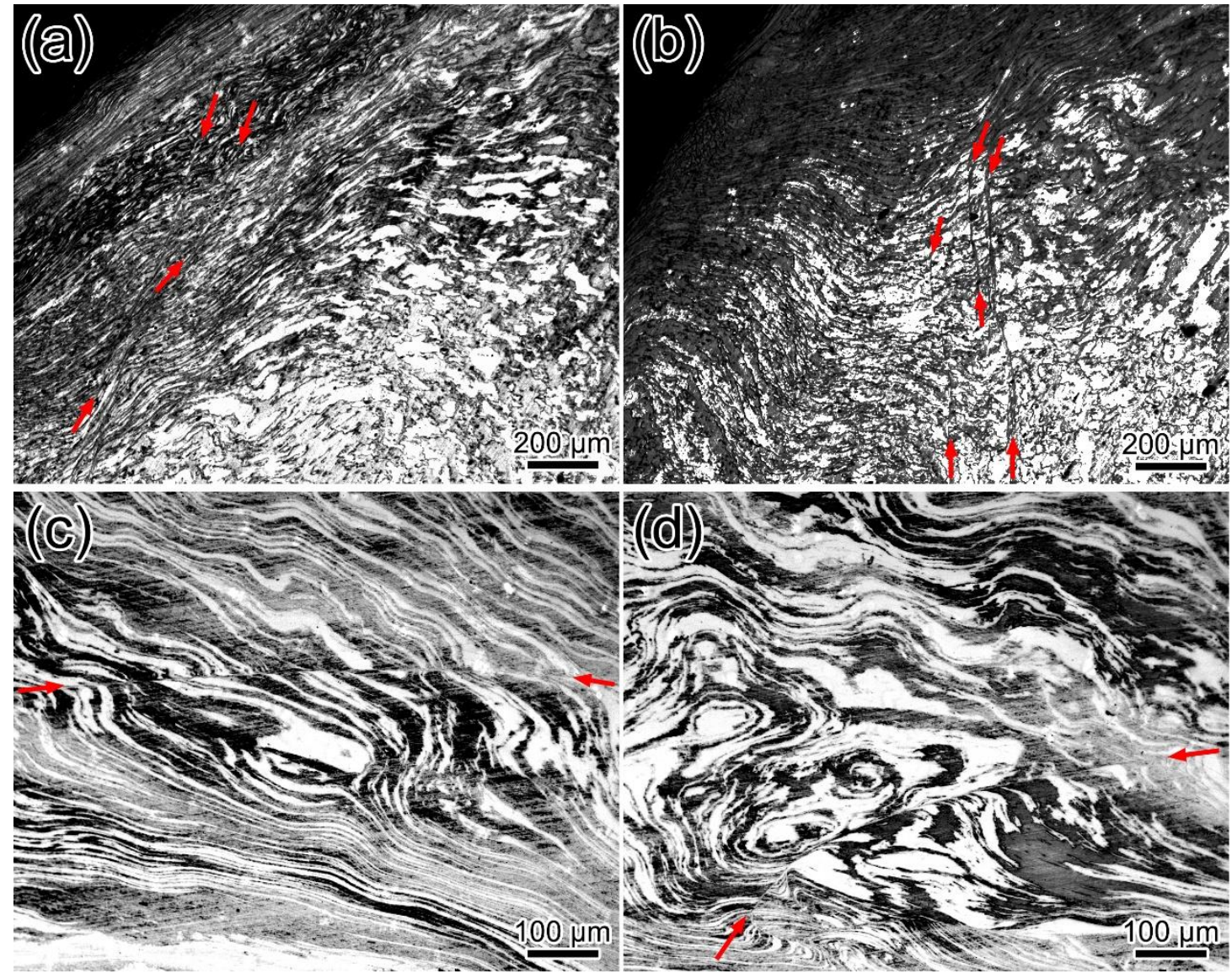

Fig. 4. Optical micrographs showing macroscopic shear strain patterns in HPT disks: (a) and

(b) are taken from the top surface of the disk processed to 3 revolutions under 6 GPa pressure;

(c) and (d) are taken from the top surface of the disk processed to 5 revolutions under $6 \mathrm{GPa}$

pressure. Red arrows mark sharp shear bands. 


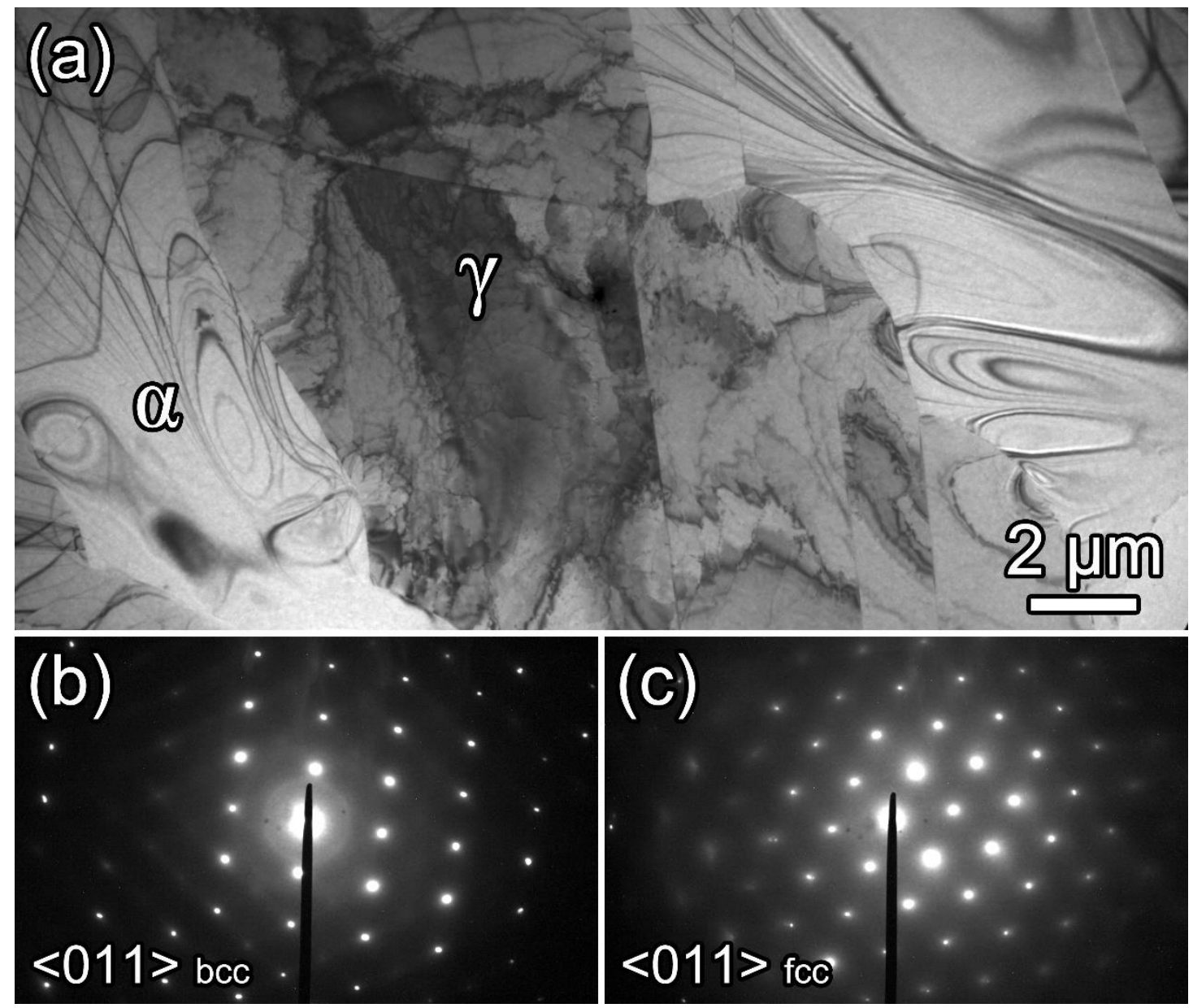

Fig. 5. (a) A TEM image showing microstructures of $\alpha$-phase and $\gamma$-phase in an as-received DP3W duplex stainless steel sample. (b) and (c) are diffraction patterns for the $\alpha$-phase-grain and $\gamma$-phase-grain labeled in (a), respectively. 


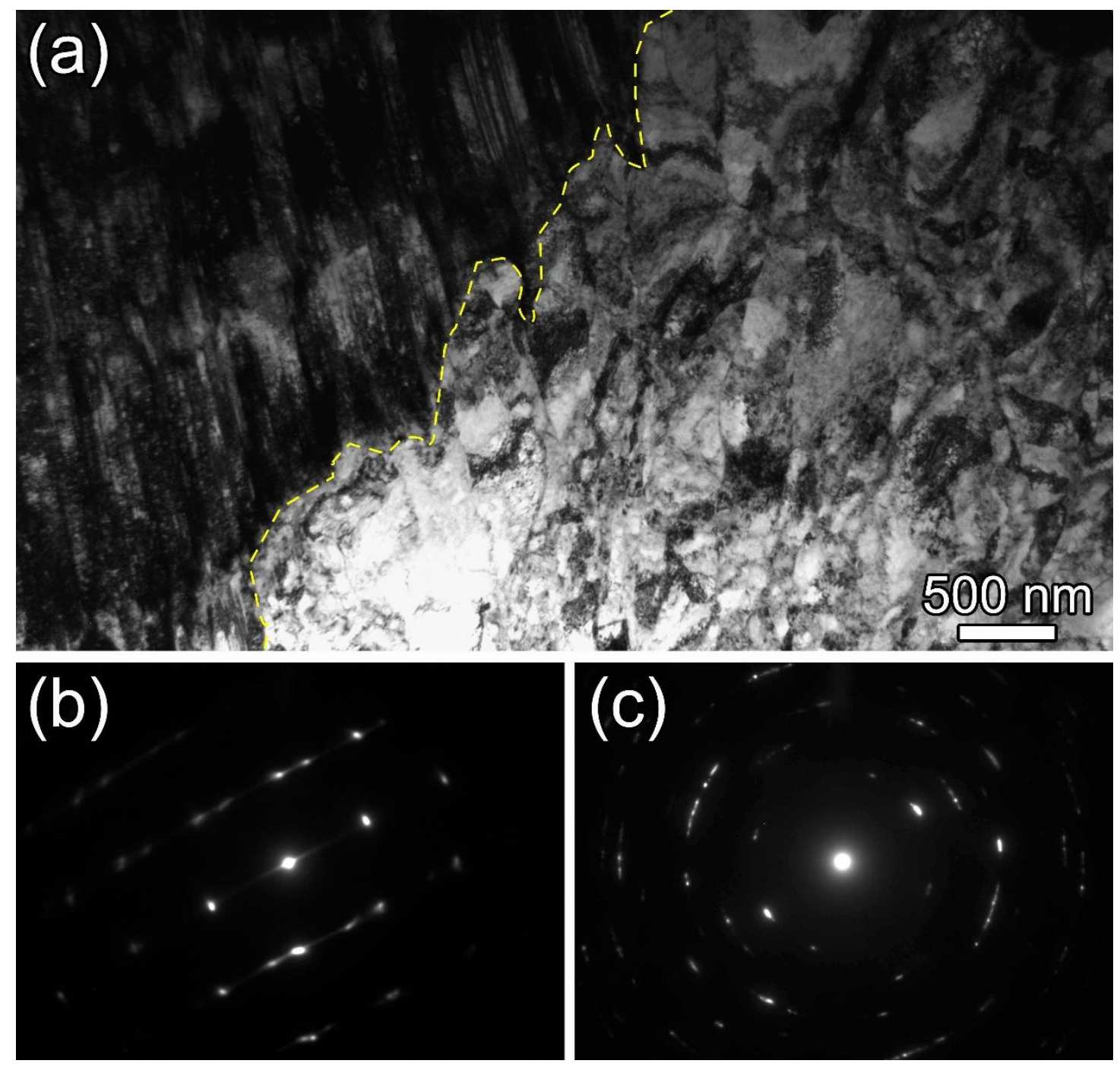

Fig. 6. (a) A TEM image showing microstructures of $\alpha$-phase and $\gamma$-phase near the center of a HPT disk processed to 1 revolution under 6 GPa pressure. (b) and (c) are diffraction patterns for the $\gamma$-phase and $\alpha$-phase, respectively. 


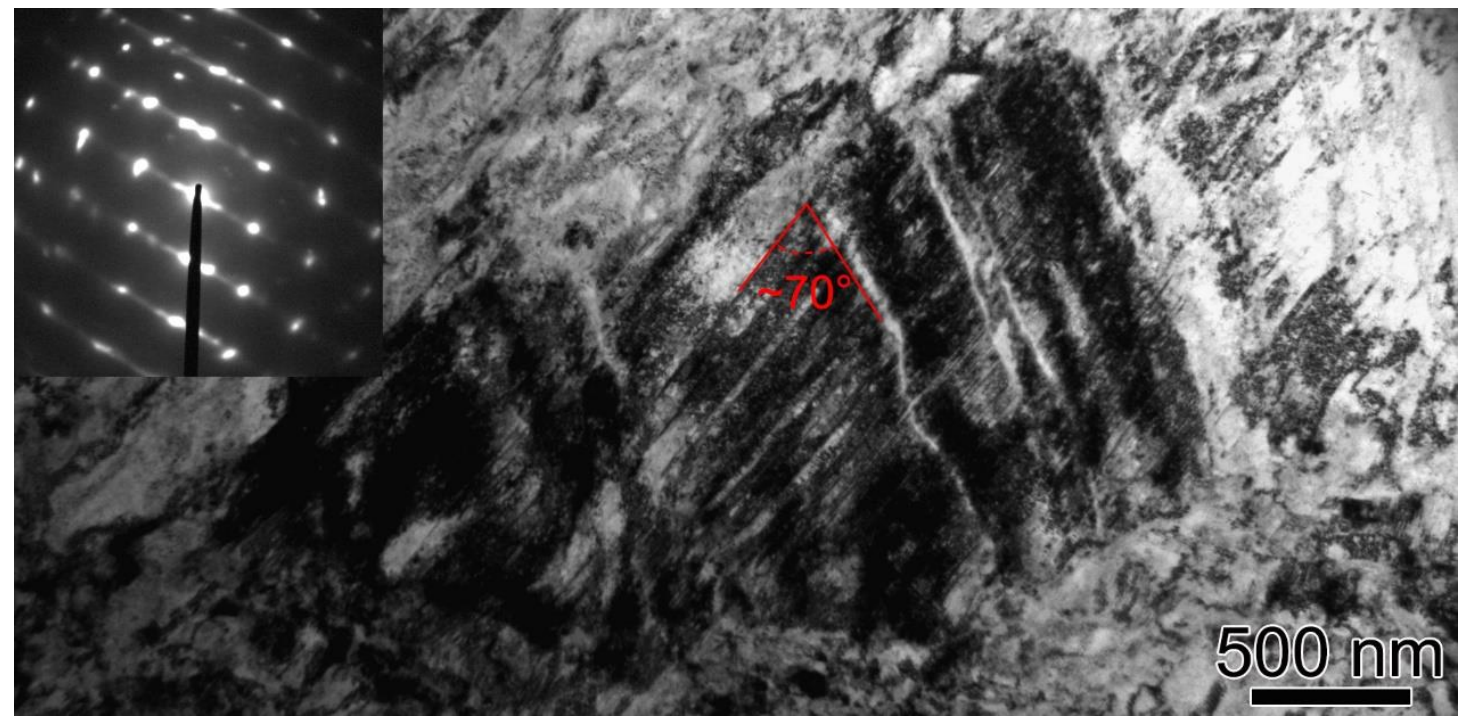

Fig. 7. A TEM image showing the grain refinement process of an austenite grain containing a high density of twins. This image was taken near the center of a HPT disk processed to 5 revolutions under 6 GPa pressure. The inset is the diffraction pattern from the austenite grain.

The angle between a white band and the $\{111\}$ twinning plane is approximately $70^{\circ}$. 

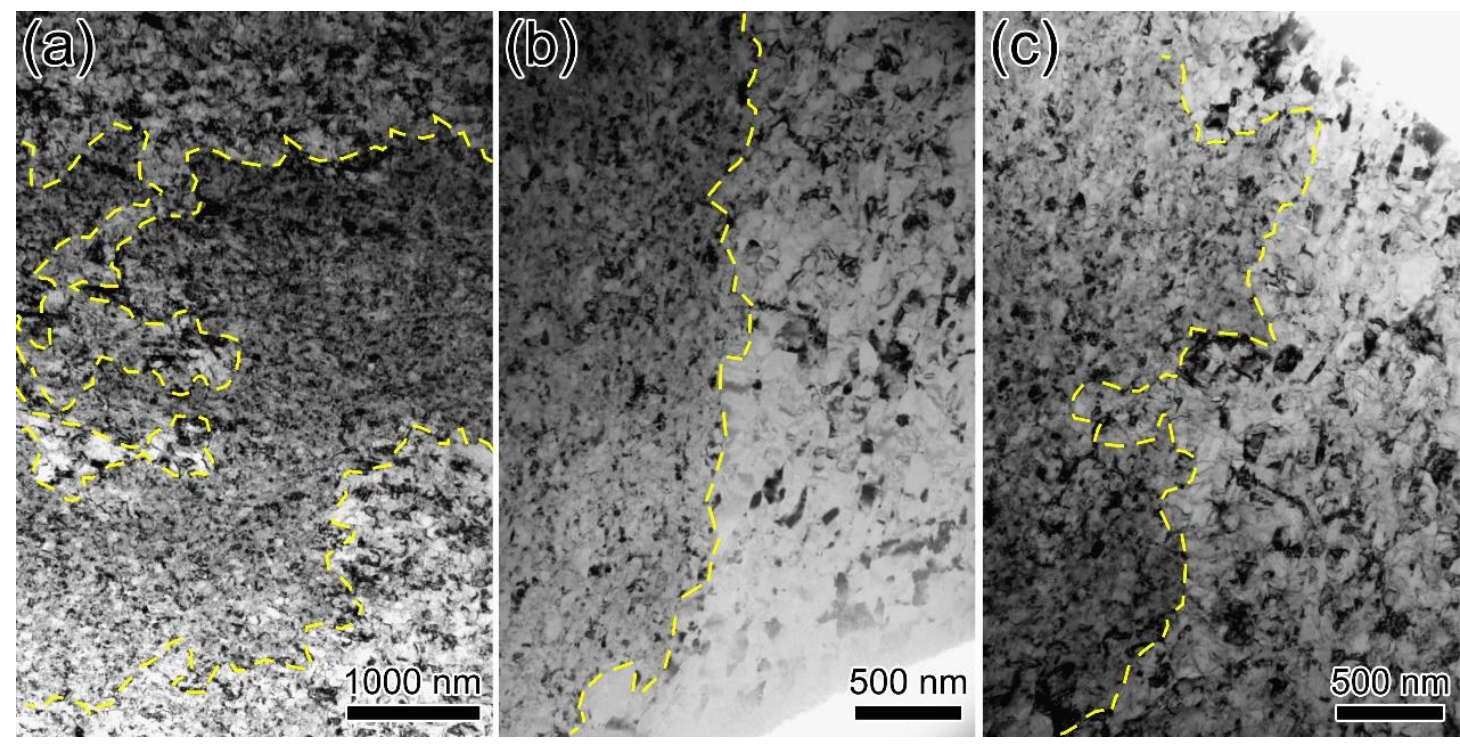

Fig. 8. TEM images showing (a) $\gamma$-phase domains of irregular shapes, (b) a comparatively straight interface between phase domains, and (c) a wavy interface between phase domains.

Yellow dashed lines trace interfaces between $\alpha$-phase and $\gamma$-phase domains. The images are taken from the edge of a HPT disk processed to 5 revolution under 6 GPa pressure. 

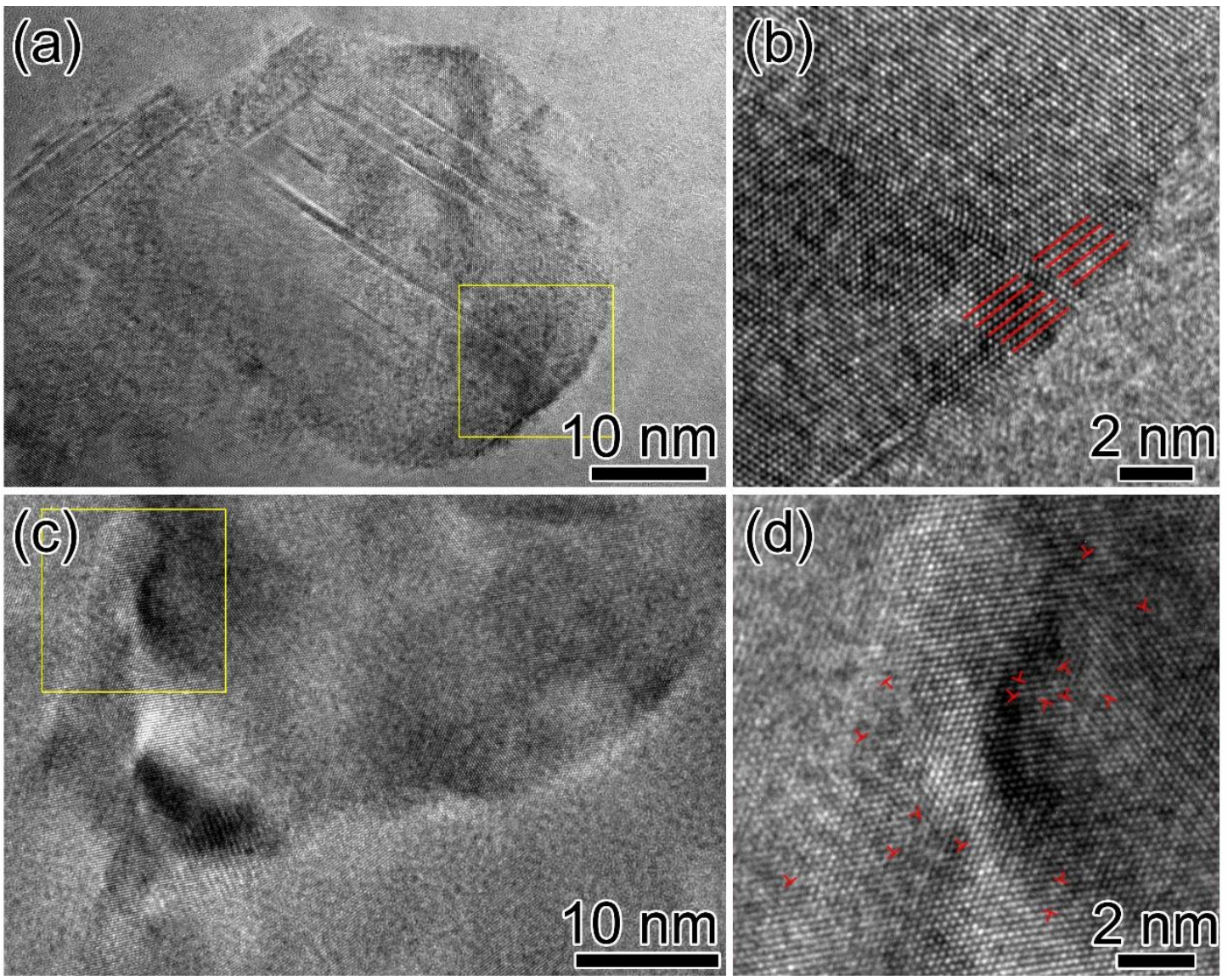

Fig. 9. High resolution TEM images showing atomic structures of grains at the edge of a HPT

disk processed to 5 revolutions under 6 GPa pressure: (a) an austenite grain viewed at [011] zone axis, (b) an enlarged image of the selected area in (a), (c) a part of a ferrite grain at [111] zone axis, and (d) an enlarged image of the selected area in (c). (Red lines trace (1111) planes inclined to an extrinsic stacking fault. A red "T" marks a dislocation component.) 